
COMMUNICATIONS

ISSN 2056-9890

\section{Crystal structure of mandipropamid}

\author{
Hyunjin Park, Jineun Kim,* Gihaeng Kang and Tae Ho
}

Kim*

Department of Chemistry and Research Institute of Natural Sciences, Gyeongsang National University, Jinju 52828, Republic of Korea. *Correspondence e-mail:

jekim@gnu.ac.kr, thkim@gnu.ac.kr

Received 31 August 2015; accepted 7 September 2015

Edited by E. R. T. Tiekink, University of Malaya, Malaysia

In the title compound, $\mathrm{C}_{23} \mathrm{H}_{22} \mathrm{ClNO}_{4}$ (systematic name: $(R S)$ 2-(4-chlorophenyl)- $N$-\{2-[3-methoxy-4-(prop-2-yn-1-yloxy)phenyl]ethyl\}-2-(prop-2-ynyloxy)acetamide), an amide fungicide, the dihedral angle between the chlorobenzene and benzene rings is $65.36(6)^{\circ}$. In the crystal, $\mathrm{N}-\mathrm{H} \cdots \mathrm{O}$ hydrogen bonds lead to zigzag supramolecular chains along the $c$ axis (glide symmetry). These are connected into layers by $\mathrm{C}-$ $\mathrm{H} \cdots \mathrm{O}$ and $\mathrm{C}-\mathrm{H} \cdots \pi$ interactions; the layers stack along the $a$ axis with no specific intermolecular interactions between them.

Keywords: crystal structure; conformation; hydrogen bonding; $\mathrm{C}-\mathrm{H} \cdots \pi$ interactions; amide fungicide.

CCDC reference: 1422569

\section{Related literature}

For information on the fungicidal properties of the title compound, see: Zhang et al. (2014). For a related crystal structure, see: Davis \& Healy (2008).

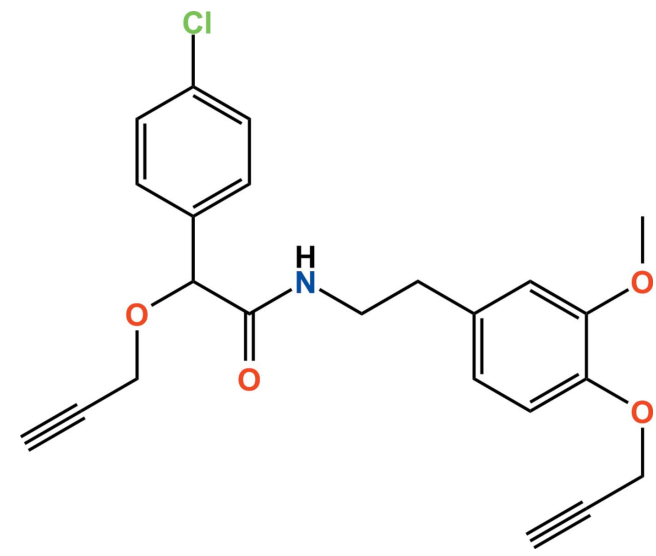

\section{Experimental}

2.1. Crystal data

$\mathrm{C}_{23} \mathrm{H}_{22} \mathrm{ClNO}_{4}$

$M_{r}=411.86$

Monoclinic, $P 2_{1} / c$

$a=26.3733(17) \AA$

$b=9.4740(6) \AA$

$c=8.4882(5) \AA$

$\beta=91.013(3)^{\circ}$

$V=2120.5(2) \AA^{3}$

$Z=4$

Mo $K \alpha$ radiation

$\mu=0.21 \mathrm{~mm}^{-1}$

$T=173 \mathrm{~K}$

$0.22 \times 0.15 \times 0.10 \mathrm{~mm}$

\subsection{Data collection}

Bruker APEXII CCD diffractometer

Absorption correction: multi-scan (SADABS; Bruker, 2013)

$T_{\min }=0.722, T_{\max }=0.746$

\subsection{Refinement}

$R\left[F^{2}>2 \sigma\left(F^{2}\right)\right]=0.052$

$w R\left(F^{2}\right)=0.131$

$S=1.04$

4885 reflections

263 parameters

$\mathrm{H}$-atom parameters constrained

$\Delta \rho_{\max }=0.82 \mathrm{e} \AA^{-3}$

$\Delta \rho_{\min }=-0.29 \mathrm{e} \AA^{-3}$

Table 1

Hydrogen-bond geometry $\left(\AA{ }^{\circ}\right)$.

$\mathrm{Cg} 2$ is the centroid of the $\mathrm{C} 14-\mathrm{C} 19$ ring.

\begin{tabular}{lllll}
\hline$D-\mathrm{H} \cdots A$ & $D-\mathrm{H}$ & $\mathrm{H} \cdots A$ & $D \cdots A$ & $D-\mathrm{H} \cdots A$ \\
\hline $\mathrm{N} 1-\mathrm{H} 1 N \cdots \mathrm{O} 2^{\mathrm{i}}$ & 0.88 & 2.04 & $2.850(2)$ & 152 \\
$\mathrm{C} 10-\mathrm{H} 10 \cdots \mathrm{O} 2^{\text {ii }}$ & 0.95 & 2.35 & $3.218(3)$ & 152 \\
$\mathrm{C} 12-\mathrm{H} 12 A \cdots \mathrm{O} 1^{\text {iii }}$ & 0.99 & 2.53 & $3.232(3)$ & 128 \\
$\mathrm{C} 20-\mathrm{H} 20 C \cdots C g 2^{\text {iv }}$ & 0.98 & 2.84 & $3.709(3)$ & 148 \\
\hline
\end{tabular}

Symmetry codes: (i) $x,-y+\frac{1}{2}, z-\frac{1}{2}$; (ii) $x,-y+\frac{3}{2}, z-\frac{1}{2}$; (iii) $x,-y+\frac{1}{2}, z+\frac{1}{2}$; (iv) $x,-y-\frac{1}{2}, z-\frac{1}{2}$.

Data collection: APEX2 (Bruker, 2013); cell refinement: SAINT (Bruker, 2013); data reduction: $S A I N T$; program(s) used to solve structure: SHELXS97 (Sheldrick, 2008); program(s) used to refine structure: SHELXL2013 (Sheldrick, 2015); molecular graphics: DIAMOND (Brandenburg, 2010); software used to prepare material for publication: SHELXTL (Sheldrick, 2008).

\title{
Acknowledgements
}

This research was supported by the Basic Science Research Program through the National Research Foundation of Korea (NRF) funded by the Ministry of Education, Science and Technology (grnat No. 2014R1A1A4A01009105).

Supporting information for this paper is available from the IUCr electronic archives (Reference: TK5382). 


\section{data reports}

\section{References}

Brandenburg, K. (2010). DIAMOND. Crystal Impact GbR, Bonn, Germany. Bruker (2013). APEX2, SAINT and SADABS. Bruker AXS Inc., Madison, Wisconsin, USA.

Davis, R. A. \& Healy, P. C. (2008). Acta Cryst. E64, o1057.
Sheldrick, G. M. (2008). Acta Cryst. A64, 112-122.

Sheldrick, G. M. (2015). Acta Cryst. C71, 3-8.

Zhang, H., Wang, X., Wang, X., Qian, M., Xu, M., Xu, H., Qi, P., Wang, Q. \& Zhuang, S. (2014). J. Sep. Sci. 37, 211-218. 


\section{supporting information}

Acta Cryst. (2015). E71, o727-o728 [doi:10.1107/S2056989015016643]

\section{Crystal structure of mandipropamid}

\section{Hyunjin Park, Jineun Kim, Gihaeng Kang and Tae Ho Kim}

\section{S1. Comment}

Mandipropamid [systematic name: $(R S)$-2-(4-chlorophenyl)- $N$-[3-methoxy-4-(prop-2-ynyloxy)phenethyl]-2-(prop-2ynyloxy)acetamide] is a the amide fungicide that is used on potato, tomato, pepper, grape, watermelon, and litchi. (Zhang et al., 2014). The dihedral angle between the chlorobenzene and the benzene rings is $65.36(6)^{\circ}$. All bond lengths and bond angles are normal and comparable to those observed in a similar crystal structure (Davis \& Healy, 2008).

In the crystal structure (Fig. 2), $\mathrm{N}-\mathrm{H} \cdots \mathrm{O}$ and $\mathrm{C}-\mathrm{H} \cdots \mathrm{O}$ hydrogen bonds and weak $\mathrm{C}-\mathrm{H} \cdots \pi$ interactions (Table 1) link adjacent molecules.

\section{S2. Experimental}

The title compound was purchased from Dr. Ehrenstorfer $\mathrm{GmbH}$. Slow evaporation of its solution in $\mathrm{CH}_{3} \mathrm{CN}_{\text {gave single }}$ crystals suitable for X-ray analysis.

\section{S3. Refinement}

All H-atoms were positioned geometrically and refined using a riding model with $\mathrm{d}(\mathrm{N}-\mathrm{H})=0.88 \AA, U_{\text {iso }}=1.2 U_{\text {eq }}(\mathrm{C})$ for $\mathrm{N}-\mathrm{H}$, and $\mathrm{d}(\mathrm{C}-\mathrm{H})=0.95-1.00 \AA, U_{\mathrm{iso}}=1.2-1.5 U_{\mathrm{eq}}(\mathrm{C})$ for $\mathrm{C}-\mathrm{H}$. Owing to poor agreement, one reflection, i.e. $\left(\begin{array}{lll}1 & 0 & 0\end{array}\right)$, was omitted from the final cycles of refinement. 


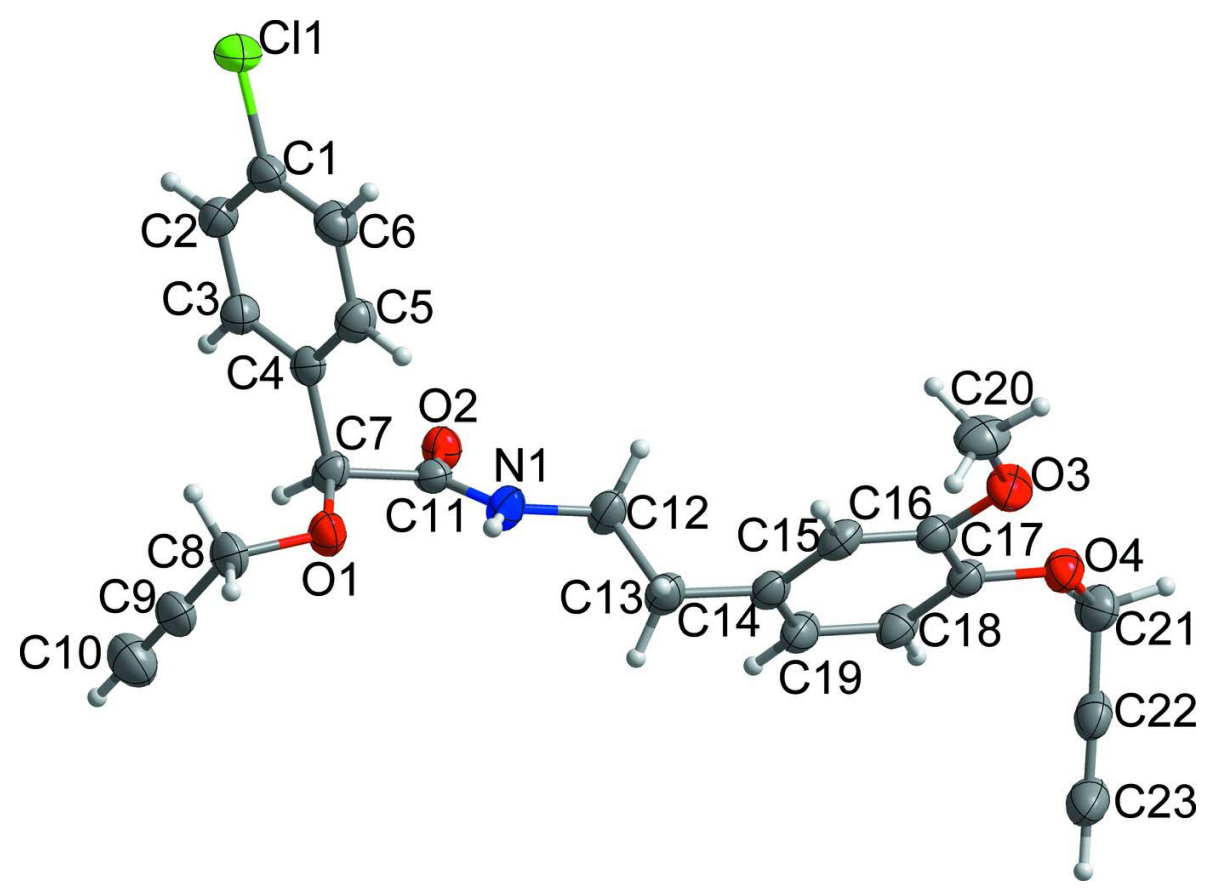

\section{Figure 1}

The molecular structure of the title compound with the atom-numbering scheme. Displacement ellipsoids are drawn at the $50 \%$ probability level. $\mathrm{H}$ atoms are shown as small spheres of arbitrary radius.

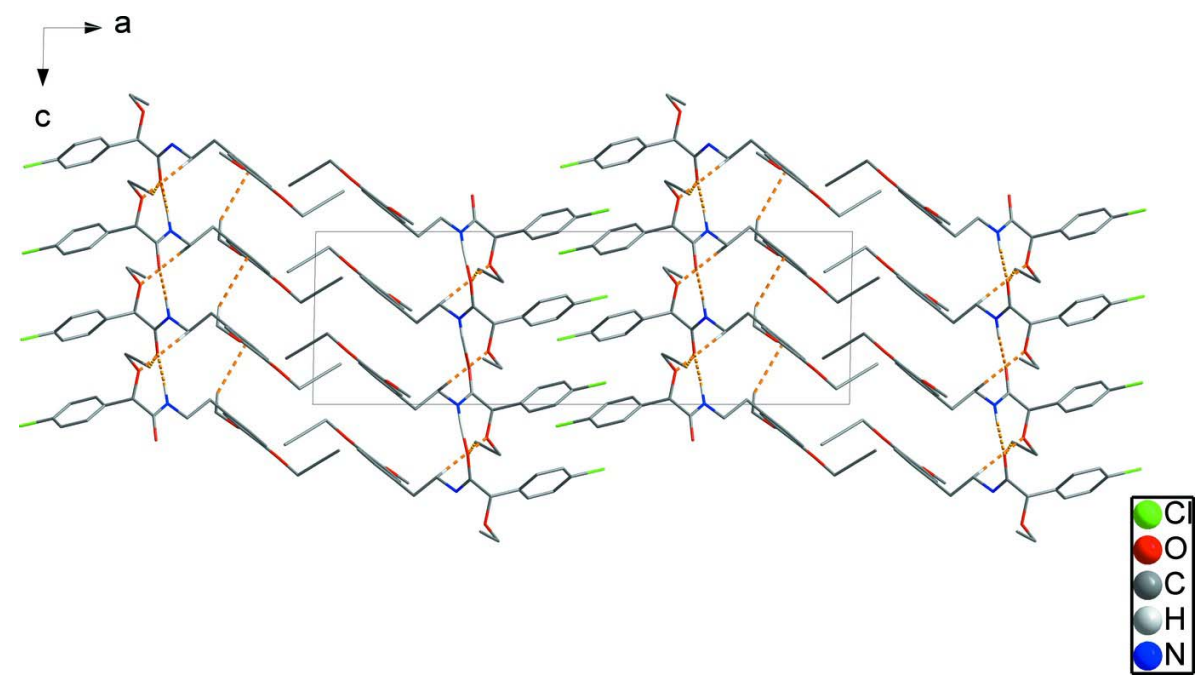

\section{Figure 2}

Crystal packing viewed along the $b$ axis. The hydrogen bonds are shown as dashed lines.

(RS)-2-(4-Chlorophenyl)-N-\{2-[3-methoxy-4-(prop-2-yn-1-yloxy)phenyl]ethyl\}-2-(prop-2-ynyloxy)acetamide

Crystal data

$\mathrm{C}_{23} \mathrm{H}_{22} \mathrm{ClNO}_{4}$

$M_{r}=411.86$

Monoclinic, $P 2{ }_{1} / c$

$a=26.3733(17) \AA$

$b=9.4740(6) \AA$

$$
\begin{aligned}
& c=8.4882(5) \AA \\
& \beta=91.013(3)^{\circ} \\
& V=2120.5(2) \AA^{3} \\
& Z=4 \\
& F(000)=864
\end{aligned}
$$


$D_{\mathrm{x}}=1.290 \mathrm{Mg} \mathrm{m}^{-3}$

Mo $K \alpha$ radiation, $\lambda=0.71073 \AA$

Cell parameters from 9957 reflections

$\theta=2.3-26.3^{\circ}$

\section{Data collection}

Bruker APEXII CCD

diffractometer

$\varphi$ and $\omega$ scans

Absorption correction: multi-scan

(SADABS; Bruker, 2013)

$T_{\min }=0.722, T_{\max }=0.746$

36727 measured reflections

\section{Refinement}

Refinement on $F^{2}$

Least-squares matrix: full

$R\left[F^{2}>2 \sigma\left(F^{2}\right)\right]=0.052$

$w R\left(F^{2}\right)=0.131$

$S=1.04$

4885 reflections

263 parameters

0 restraints $\mu=0.21 \mathrm{~mm}^{-1}$

$T=173 \mathrm{~K}$

Plate, colourless

$0.22 \times 0.15 \times 0.10 \mathrm{~mm}$

4885 independent reflections

3686 reflections with $I>2 \sigma(I)$

$R_{\text {int }}=0.039$

$\theta_{\text {max }}=27.6^{\circ}, \theta_{\text {min }}=2.3^{\circ}$

$h=-33 \rightarrow 34$

$k=-12 \rightarrow 12$

$l=-11 \rightarrow 10$

Hydrogen site location: inferred from neighbouring sites

$\mathrm{H}$-atom parameters constrained

$w=1 /\left[\sigma^{2}\left(F_{\mathrm{o}}{ }^{2}\right)+(0.0444 P)^{2}+1.677 P\right]$

where $P=\left(F_{\mathrm{o}}{ }^{2}+2 F_{\mathrm{c}}{ }^{2}\right) / 3$

$(\Delta / \sigma)_{\max }=0.001$

$\Delta \rho_{\max }=0.82 \mathrm{e} \AA^{-3}$

$\Delta \rho_{\min }=-0.29$ e $\AA^{-3}$

Special details

Geometry. All e.s.d.'s (except the e.s.d. in the dihedral angle between two 1.s. planes) are estimated using the full covariance matrix. The cell e.s.d.'s are taken into account individually in the estimation of e.s.d.'s in distances, angles and torsion angles; correlations between e.s.d.'s in cell parameters are only used when they are defined by crystal symmetry. An approximate (isotropic) treatment of cell e.s.d.'s is used for estimating e.s.d.'s involving 1.s. planes.

Fractional atomic coordinates and isotropic or equivalent isotropic displacement parameters $\left(\AA^{2}\right)$

\begin{tabular}{lllll}
\hline & $x$ & $y$ & $z$ & $U_{\text {iso }} / U_{\text {eq }}$ \\
\hline C11 & $0.45595(2)$ & $0.22978(7)$ & $0.12537(9)$ & $0.0543(2)$ \\
O1 & $0.67498(6)$ & $0.42193(16)$ & $-0.19373(17)$ & $0.0376(4)$ \\
O2 & $0.70934(5)$ & $0.38856(15)$ & $0.20630(16)$ & $0.0332(3)$ \\
O3 & $0.86165(6)$ & $-0.36494(16)$ & $0.1328(2)$ & $0.0442(4)$ \\
O4 & $0.93566(5)$ & $-0.26941(15)$ & $0.30322(18)$ & $0.0383(4)$ \\
N1 & $0.72949(6)$ & $0.25526(19)$ & $-0.0044(2)$ & $0.0341(4)$ \\
H1N & 0.7239 & 0.2407 & -0.1056 & $0.041^{*}$ \\
C1 & $0.51676(8)$ & $0.2878(2)$ & $0.0816(3)$ & $0.0355(5)$ \\
C2 & $0.53176(8)$ & $0.4194(2)$ & $0.1302(3)$ & $0.0375(5)$ \\
H2 & 0.5094 & 0.4775 & 0.1883 & $0.045^{*}$ \\
C3 & $0.57995(8)$ & $0.4671(2)$ & $0.0936(2)$ & $0.0341(5)$ \\
H3 & 0.5908 & 0.5576 & 0.1286 & $0.041^{*}$ \\
C4 & $0.61212(7)$ & $0.3842(2)$ & $0.0069(2)$ & $0.0298(4)$ \\
C5 & $0.59606(8)$ & $0.2516(2)$ & $-0.0420(3)$ & $0.0375(5)$ \\
H5 & 0.6179 & 0.1942 & -0.1025 & $0.045^{*}$ \\
C6 & $0.54833(9)$ & $0.2019(2)$ & $-0.0035(3)$ & $0.0404(5)$ \\
H6 & 0.5376 & 0.1103 & -0.0352 & $0.048^{*}$ \\
C7 & $0.66491(7)$ & $0.4385(2)$ & $-0.0328(2)$ & $0.0315(4)$
\end{tabular}




$\begin{array}{lllll}\text { H7 } & 0.6672 & 0.5409 & -0.0051 & 0.038^{*} \\ \text { C8 } & 0.64981(9) & 0.5251(2) & -0.2903(3) & 0.0417(5) \\ \text { H8A } & 0.6130 & 0.5220 & -0.2700 & 0.050^{*} \\ \text { H8B } & 0.6547 & 0.5010 & -0.4025 & 0.050^{*} \\ \text { C9 } & 0.66839(9) & 0.6681(3) & -0.2614(3) & 0.0446(6) \\ \text { C10 } & 0.68291(11) & 0.7840(3) & -0.2365(3) & 0.0575(7) \\ \text { H10 } & 0.6946 & 0.8773 & -0.2164 & 0.069^{*} \\ \text { C11 } & 0.70398(7) & 0.3566(2) & 0.0662(2) & 0.0291(4) \\ \text { C12 } & 0.76667(8) & 0.1662(3) & 0.0785(3) & 0.0380(5) \\ \text { H12A } & 0.7492 & 0.0829 & 0.1222 & 0.046^{*} \\ \text { H12B } & 0.7817 & 0.2200 & 0.1678 & 0.046^{*} \\ \text { C13 } & 0.80847(8) & 0.1170(3) & -0.0268(3) & 0.0387(5) \\ \text { H13A } & 0.7936 & 0.0687 & -0.1202 & 0.046^{*} \\ \text { H13B } & 0.8280 & 0.1994 & -0.0639 & 0.046^{*} \\ \text { C14 } & 0.84327(8) & 0.0174(2) & 0.0610(2) & 0.0346(5) \\ \text { C15 } & 0.83530(8) & -0.1280(2) & 0.0534(3) & 0.0355(5) \\ \text { H15 } & 0.8081 & -0.1637 & -0.0096 & 0.043^{*} \\ \text { C16 } & 0.86616(8) & -0.2208(2) & 0.1356(3) & 0.0343(5) \\ \text { C17 } & 0.90637(7) & -0.1685(2) & 0.2301(2) & 0.0329(5) \\ \text { C18 } & 0.91396(8) & -0.0250(2) & 0.2401(3) & 0.0376(5) \\ \text { H18 } & 0.9407 & 0.0113 & 0.3046 & 0.045^{*} \\ \text { C19 } & 0.88242(8) & 0.0672(2) & 0.1556(3) & 0.0389(5) \\ \text { H19 } & 0.8880 & 0.1661 & 0.1633 & 0.047^{*} \\ \text { C20 } & 0.81997(9) & -0.4224(3) & 0.0456(3) & 0.0503(6) \\ \text { H20A } & 0.7882 & -0.3864 & 0.0881 & 0.075^{*} \\ \text { H20B } & 0.8206 & -0.5256 & 0.0538 & 0.075^{*} \\ \text { H20C } & 0.8225 & -0.3948 & -0.0653 & 0.075^{*} \\ \text { C21 } & 0.97493(9) & -0.2226(3) & 0.4095(3) & 0.0428(5) \\ \text { H21A } & 0.9884 & -0.3049 & 0.4687 & 0.051^{*} \\ \text { H21B } & 0.9603 & -0.1563 & 0.4864 & 0.051^{*} \\ \text { C22 } & 1.01689(8) & -0.1525(2) & 0.3292(3) & 0.0417(5) \\ \text { C23 } & 1.05017(10) & -0.0922(3) & 0.2672(4) & 0.0542(7) \\ \text { H23 } & 1.0770 & -0.0435 & 0.2173 & 0.065^{*}\end{array}$

Atomic displacement parameters $\left(\AA^{2}\right)$

\begin{tabular}{lllllll}
\hline & $U^{11}$ & $U^{22}$ & $U^{33}$ & $U^{12}$ & $U^{13}$ & $U^{23}$ \\
\hline C11 & $0.0349(3)$ & $0.0530(4)$ & $0.0753(5)$ & $-0.0051(3)$ & $0.0082(3)$ & $0.0229(3)$ \\
O1 & $0.0426(9)$ & $0.0383(8)$ & $0.0318(8)$ & $0.0099(7)$ & $0.0019(6)$ & $0.0045(6)$ \\
O2 & $0.0363(8)$ & $0.0359(8)$ & $0.0272(8)$ & $0.0032(6)$ & $-0.0033(6)$ & $-0.0002(6)$ \\
O3 & $0.0406(9)$ & $0.0346(8)$ & $0.0572(10)$ & $-0.0052(7)$ & $-0.0040(8)$ & $-0.0044(7)$ \\
O4 & $0.0323(8)$ & $0.0362(8)$ & $0.0462(9)$ & $0.0008(6)$ & $-0.0047(7)$ & $0.0013(7)$ \\
N1 & $0.0321(9)$ & $0.0464(10)$ & $0.0238(9)$ & $0.0094(8)$ & $-0.0022(7)$ & $0.0002(8)$ \\
C1 & $0.0289(10)$ & $0.0399(12)$ & $0.0376(12)$ & $-0.0010(9)$ & $-0.0011(9)$ & $0.0130(10)$ \\
C2 & $0.0335(11)$ & $0.0385(12)$ & $0.0406(12)$ & $0.0072(9)$ & $0.0060(9)$ & $0.0049(10)$ \\
C3 & $0.0329(11)$ & $0.0322(11)$ & $0.0370(12)$ & $0.0015(9)$ & $-0.0007(9)$ & $0.0025(9)$ \\
C4 & $0.0278(10)$ & $0.0342(10)$ & $0.0272(10)$ & $0.0023(8)$ & $-0.0040(8)$ & $0.0065(8)$ \\
C5 & $0.0370(12)$ & $0.0410(12)$ & $0.0347(12)$ & $0.0057(9)$ & $0.0025(9)$ & $-0.0018(9)$
\end{tabular}


supporting information

$\begin{array}{lllllll}\text { C6 } & 0.0408(12) & 0.0361(12) & 0.0440(13) & -0.0038(10) & -0.0043(10) & 0.0021(10) \\ \text { C7 } & 0.0285(10) & 0.0390(11) & 0.0270(10) & 0.0053(8) & -0.0022(8) & -0.0002(9) \\ \text { C8 } & 0.0417(12) & 0.0439(13) & 0.0394(13) & 0.0066(10) & -0.0042(10) & 0.0133(10) \\ \text { C9 } & 0.0429(13) & 0.0469(14) & 0.0439(14) & 0.0045(11) & -0.0002(10) & 0.0175(11) \\ \text { C10 } & 0.0650(18) & 0.0500(16) & 0.0574(17) & -0.0014(13) & -0.0046(14) & 0.0125(13) \\ \text { C11 } & 0.0238(9) & 0.0344(11) & 0.0290(11) & -0.0015(8) & 0.0015(8) & 0.0046(8) \\ \text { C12 } & 0.0341(11) & 0.0478(13) & 0.0321(12) & 0.0116(10) & 0.0027(9) & 0.0022(10) \\ \text { C13 } & 0.0334(11) & 0.0467(13) & 0.0362(12) & 0.0098(10) & 0.0053(9) & 0.0029(10) \\ \text { C14 } & 0.0282(10) & 0.0419(12) & 0.0339(11) & 0.0056(9) & 0.0063(8) & -0.0003(9) \\ \text { C15 } & 0.0260(10) & 0.0448(12) & 0.0355(12) & 0.0007(9) & 0.0004(9) & -0.0061(10) \\ \text { C16 } & 0.0300(10) & 0.0342(11) & 0.0389(12) & -0.0003(9) & 0.0049(9) & -0.0039(9) \\ \text { C17 } & 0.0262(10) & 0.0369(11) & 0.0356(11) & 0.0040(8) & 0.0036(8) & -0.0006(9) \\ \text { C18 } & 0.0273(10) & 0.0384(11) & 0.0471(13) & -0.0005(9) & -0.0014(9) & -0.0071(10) \\ \text { C19 } & 0.0295(11) & 0.0331(11) & 0.0541(14) & 0.0025(9) & 0.0034(10) & -0.0022(10) \\ \text { C20 } & 0.0419(13) & 0.0477(14) & 0.0611(16) & -0.0150(11) & 0.0014(12) & -0.0080(12) \\ \text { C21 } & 0.0380(12) & 0.0478(13) & 0.0426(13) & 0.0008(10) & -0.0064(10) & 0.0044(11) \\ \text { C22 } & 0.0317(11) & 0.0387(12) & 0.0542(14) & 0.0063(10) & -0.0086(10) & 0.0004(11) \\ \text { C23 } & 0.0346(13) & 0.0445(14) & 0.083(2) & 0.0032(11) & -0.0008(13) & 0.0063(14) \\ & & & & & \end{array}$

Geometric parameters $\left(\AA,{ }^{\circ}\right)$

\begin{tabular}{llll}
\hline $\mathrm{C} 11-\mathrm{C} 1$ & $1.742(2)$ & $\mathrm{C} 8-\mathrm{H} 8 \mathrm{~B}$ & 0.9900 \\
$\mathrm{O} 1-\mathrm{C} 7$ & $1.405(2)$ & $\mathrm{C} 9-\mathrm{C} 10$ & $1.181(4)$ \\
$\mathrm{O} 1-\mathrm{C} 8$ & $1.432(2)$ & $\mathrm{C} 10-\mathrm{H} 10$ & 0.9500 \\
$\mathrm{O} 2-\mathrm{C} 11$ & $1.232(2)$ & $\mathrm{C} 12-\mathrm{C} 13$ & $1.506(3)$ \\
$\mathrm{O} 3-\mathrm{C} 16$ & $1.371(3)$ & $\mathrm{C} 12-\mathrm{H} 12 \mathrm{~A}$ & 0.9900 \\
$\mathrm{O} 3-\mathrm{C} 20$ & $1.423(3)$ & $\mathrm{C} 12-\mathrm{H} 12 \mathrm{~B}$ & 0.9900 \\
$\mathrm{O} 4-\mathrm{C} 17$ & $1.371(2)$ & $\mathrm{C} 13-\mathrm{C} 14$ & $1.505(3)$ \\
$\mathrm{O} 4-\mathrm{C} 21$ & $1.432(3)$ & $\mathrm{C} 13-\mathrm{H} 13 \mathrm{~A}$ & 0.9900 \\
$\mathrm{~N} 1-\mathrm{C} 11$ & $1.323(3)$ & $\mathrm{C} 13-\mathrm{H} 13 \mathrm{~B}$ & 0.9900 \\
$\mathrm{~N} 1-\mathrm{C} 12$ & $1.464(3)$ & $\mathrm{C} 14-\mathrm{C} 19$ & $1.380(3)$ \\
$\mathrm{N} 1-\mathrm{H} 1 \mathrm{~N}$ & 0.8800 & $\mathrm{C} 14-\mathrm{C} 15$ & $1.394(3)$ \\
$\mathrm{C} 1-\mathrm{C} 2$ & $1.369(3)$ & $\mathrm{C} 15-\mathrm{C} 16$ & $1.379(3)$ \\
$\mathrm{C} 1-\mathrm{C} 6$ & $1.378(3)$ & $\mathrm{C} 15-\mathrm{H} 15$ & 0.9500 \\
$\mathrm{C} 2-\mathrm{C} 3$ & $1.389(3)$ & $\mathrm{C} 16-\mathrm{C} 17$ & $1.409(3)$ \\
$\mathrm{C} 2-\mathrm{H} 2$ & 0.9500 & $\mathrm{C} 17-\mathrm{C} 18$ & $1.376(3)$ \\
$\mathrm{C} 3-\mathrm{C} 4$ & $1.379(3)$ & $\mathrm{C} 18-\mathrm{C} 19$ & $1.396(3)$ \\
$\mathrm{C} 3-\mathrm{H} 3$ & $\mathrm{C} 18-\mathrm{H} 18$ & 0.9500 \\
$\mathrm{C} 4-\mathrm{C} 5$ & 1.9500 & $\mathrm{C} 19-\mathrm{H} 19$ & 0.9500 \\
$\mathrm{C} 4-\mathrm{C} 7$ & $\mathrm{C} 20-\mathrm{H} 20 \mathrm{~A}$ & 0.9800 \\
$\mathrm{C} 5-\mathrm{C} 6$ & $1.528(3)$ & $\mathrm{C} 20-\mathrm{H} 20 \mathrm{~B}$ & 0.9800 \\
$\mathrm{C} 5-\mathrm{H} 5$ & $1.388(3)$ & $\mathrm{C} 20-\mathrm{H} 20 \mathrm{C}$ & 0.9800 \\
$\mathrm{C} 6-\mathrm{H} 6$ & 0.9500 & $\mathrm{C} 21-\mathrm{C} 22$ & $1.469(3)$ \\
$\mathrm{C} 7-\mathrm{C} 11$ & 0.9500 & $\mathrm{C} 21-\mathrm{H} 21 \mathrm{~A}$ & 0.9900 \\
$\mathrm{C} 7-\mathrm{H} 7$ & $1.529(3)$ & $\mathrm{C} 21-\mathrm{H} 21 \mathrm{~B}$ & 0.9900 \\
$\mathrm{C} 8-\mathrm{C} 9$ & 1.0000 & $\mathrm{C} 22-\mathrm{C} 23$ & $1.179(3)$ \\
$\mathrm{C} 8-\mathrm{H} 8 \mathrm{~A}$ & $1.460(4)$ & & 0.9500 \\
& 0.9900 & &
\end{tabular}


$\mathrm{C} 7-\mathrm{O} 1-\mathrm{C} 8$

$\mathrm{C} 16-\mathrm{O} 3-\mathrm{C} 20$

$\mathrm{C} 17-\mathrm{O} 4-\mathrm{C} 21$

$\mathrm{C} 11-\mathrm{N} 1-\mathrm{C} 12$

$\mathrm{C} 11-\mathrm{N} 1-\mathrm{H} 1 \mathrm{~N}$

$\mathrm{C} 12-\mathrm{N} 1-\mathrm{H} 1 \mathrm{~N}$

$\mathrm{C} 2-\mathrm{C} 1-\mathrm{C} 6$

$\mathrm{C} 2-\mathrm{C} 1-\mathrm{C} 11$

C6- $\mathrm{C} 1-\mathrm{Cl} 1$

$\mathrm{C} 1-\mathrm{C} 2-\mathrm{C} 3$

$\mathrm{C} 1-\mathrm{C} 2-\mathrm{H} 2$

$\mathrm{C} 3-\mathrm{C} 2-\mathrm{H} 2$

$\mathrm{C} 4-\mathrm{C} 3-\mathrm{C} 2$

$\mathrm{C} 4-\mathrm{C} 3-\mathrm{H} 3$

$\mathrm{C} 2-\mathrm{C} 3-\mathrm{H} 3$

$\mathrm{C} 3-\mathrm{C} 4-\mathrm{C} 5$

$\mathrm{C} 3-\mathrm{C} 4-\mathrm{C} 7$

$\mathrm{C} 5-\mathrm{C} 4-\mathrm{C} 7$

$\mathrm{C} 4-\mathrm{C} 5-\mathrm{C} 6$

$\mathrm{C} 4-\mathrm{C} 5-\mathrm{H} 5$

$\mathrm{C} 6-\mathrm{C} 5-\mathrm{H} 5$

$\mathrm{C} 1-\mathrm{C} 6-\mathrm{C} 5$

C1-C6- 66

$\mathrm{C} 5-\mathrm{C} 6-\mathrm{H} 6$

$\mathrm{O} 1-\mathrm{C} 7-\mathrm{C} 4$

$\mathrm{O} 1-\mathrm{C} 7-\mathrm{C} 11$

$\mathrm{C} 4-\mathrm{C} 7-\mathrm{C} 11$

$\mathrm{O} 1-\mathrm{C} 7-\mathrm{H} 7$

$\mathrm{C} 4-\mathrm{C} 7-\mathrm{H} 7$

$\mathrm{C} 11-\mathrm{C} 7-\mathrm{H} 7$

$\mathrm{O} 1-\mathrm{C} 8-\mathrm{C} 9$

$\mathrm{O} 1-\mathrm{C} 8-\mathrm{H} 8 \mathrm{~A}$

$\mathrm{C} 9-\mathrm{C} 8-\mathrm{H} 8 \mathrm{~A}$

$\mathrm{O} 1-\mathrm{C} 8-\mathrm{H} 8 \mathrm{~B}$

C9- $\mathrm{C} 8-\mathrm{H} 8 \mathrm{~B}$

$\mathrm{H} 8 \mathrm{~A}-\mathrm{C} 8-\mathrm{H} 8 \mathrm{~B}$

$\mathrm{C} 10-\mathrm{C} 9-\mathrm{C} 8$

C9- $\mathrm{C} 10-\mathrm{H} 10$

$\mathrm{O} 2-\mathrm{C} 11-\mathrm{N} 1$

$\mathrm{O} 2-\mathrm{C} 11-\mathrm{C} 7$

$\mathrm{N} 1-\mathrm{C} 11-\mathrm{C} 7$

$\mathrm{N} 1-\mathrm{C} 12-\mathrm{C} 13$

$\mathrm{N} 1-\mathrm{C} 12-\mathrm{H} 12 \mathrm{~A}$

$\mathrm{C} 6-\mathrm{C} 1-\mathrm{C} 2-\mathrm{C} 3$

$\mathrm{C} 11-\mathrm{C} 1-\mathrm{C} 2-\mathrm{C} 3$

$\mathrm{C} 1-\mathrm{C} 2-\mathrm{C} 3-\mathrm{C} 4$

$\mathrm{C} 2-\mathrm{C} 3-\mathrm{C} 4-\mathrm{C} 5$
$112.75(16)$

$117.13(18)$

$117.70(17)$

$122.83(17)$

118.6

118.6

$121.4(2)$

119.05 (17)

$119.50(18)$

119.3 (2)

120.3

120.3

120.5 (2)

119.7

119.7

119.24 (19)

$119.81(19)$

120.94 (19)

120.7 (2)

119.7

119.7

118.8 (2)

120.6

120.6

$111.42(16)$

$109.91(16)$

$108.39(16)$

109.0

109.0

109.0

112.75 (19)

109.0

109.0

109.0

109.0

107.8

$179.0(3)$

180.0

$124.40(18)$

$118.06(18)$

$117.53(17)$

112.59 (17)

109.1

$-0.3(3)$

$-179.05(17)$

$1.2(3)$

-0.7 (3)
$\mathrm{C} 13-\mathrm{C} 12-\mathrm{H} 12 \mathrm{~A}$

N1-C12-H12B

$\mathrm{C} 13-\mathrm{C} 12-\mathrm{H} 12 \mathrm{~B}$

$\mathrm{H} 12 \mathrm{~A}-\mathrm{C} 12-\mathrm{H} 12 \mathrm{~B}$

$\mathrm{C} 14-\mathrm{C} 13-\mathrm{C} 12$

C14-C13-H13A

C12-C13-H13A

C14-C13-H13B

C12-C13-H13B

H13A-C13-H13B

C19-C14-C15

$\mathrm{C} 19-\mathrm{C} 14-\mathrm{C} 13$

$\mathrm{C} 15-\mathrm{C} 14-\mathrm{C} 13$

C16-C15-C14

C16-C15-H15

C14-C15-H15

$\mathrm{O} 3-\mathrm{C} 16-\mathrm{C} 15$

$\mathrm{O} 3-\mathrm{C} 16-\mathrm{C} 17$

$\mathrm{C} 15-\mathrm{C} 16-\mathrm{C} 17$

$\mathrm{O} 4-\mathrm{C} 17-\mathrm{C} 18$

$\mathrm{O} 4-\mathrm{C} 17-\mathrm{C} 16$

$\mathrm{C} 18-\mathrm{C} 17-\mathrm{C} 16$

C17-C18-C19

C17-C18-H18

C19-C18-H18

$\mathrm{C} 14-\mathrm{C} 19-\mathrm{C} 18$

C14-C19-H19

C18-C19-H19

$\mathrm{O} 3-\mathrm{C} 20-\mathrm{H} 20 \mathrm{~A}$

$\mathrm{O} 3-\mathrm{C} 20-\mathrm{H} 20 \mathrm{~B}$

$\mathrm{H} 20 \mathrm{~A}-\mathrm{C} 20-\mathrm{H} 20 \mathrm{~B}$

$\mathrm{O} 3-\mathrm{C} 20-\mathrm{H} 20 \mathrm{C}$

$\mathrm{H} 20 \mathrm{~A}-\mathrm{C} 20-\mathrm{H} 20 \mathrm{C}$

$\mathrm{H} 20 \mathrm{~B}-\mathrm{C} 20-\mathrm{H} 20 \mathrm{C}$

$\mathrm{O} 4-\mathrm{C} 21-\mathrm{C} 22$

$\mathrm{O} 4-\mathrm{C} 21-\mathrm{H} 21 \mathrm{~A}$

$\mathrm{C} 22-\mathrm{C} 21-\mathrm{H} 21 \mathrm{~A}$

$\mathrm{O} 4-\mathrm{C} 21-\mathrm{H} 21 \mathrm{~B}$

$\mathrm{C} 22-\mathrm{C} 21-\mathrm{H} 21 \mathrm{~B}$

$\mathrm{H} 21 \mathrm{~A}-\mathrm{C} 21-\mathrm{H} 21 \mathrm{~B}$

$\mathrm{C} 23-\mathrm{C} 22-\mathrm{C} 21$

$\mathrm{C} 22-\mathrm{C} 23-\mathrm{H} 23$

$\mathrm{C} 11-\mathrm{N} 1-\mathrm{C} 12-\mathrm{C} 13$

$\mathrm{N} 1-\mathrm{C} 12-\mathrm{C} 13-\mathrm{C} 14$

$\mathrm{C} 12-\mathrm{C} 13-\mathrm{C} 14-\mathrm{C} 19$

$\mathrm{C} 12-\mathrm{C} 13-\mathrm{C} 14-\mathrm{C} 15$
109.1

109.1

109.1

107.8

110.27 (18)

109.6

109.6

109.6

109.6

108.1

118.4 (2)

$121.1(2)$

$120.4(2)$

$121.3(2)$

119.4

119.4

125.2 (2)

115.09 (19)

119.7 (2)

125.51 (19)

$115.16(18)$

119.3 (2)

120.1 (2)

119.9

119.9

$121.2(2)$

119.4

119.4

109.5

109.5

109.5

109.5

109.5

109.5

112.97 (19)

109.0

109.0

109.0

109.0

107.8

177.8 (3)

180.0

$-149.7(2)$

-175.73 (19)

-84.1 (3)

$93.3(3)$ 


\section{supporting information}

$\begin{array}{ll}\mathrm{C} 2-\mathrm{C} 3-\mathrm{C} 4-\mathrm{C} 7 & 179.77(19) \\ \mathrm{C} 3-\mathrm{C} 4-\mathrm{C} 5-\mathrm{C} 6 & -0.5(3) \\ \mathrm{C} 7-\mathrm{C} 4-\mathrm{C} 5-\mathrm{C} 6 & 178.97(19) \\ \mathrm{C} 2-\mathrm{C} 1-\mathrm{C} 6-\mathrm{C} 5 & -0.9(3) \\ \mathrm{C} 11-\mathrm{C} 1-\mathrm{C} 6-\mathrm{C} 5 & 177.82(17) \\ \mathrm{C} 4-\mathrm{C} 5-\mathrm{C} 6-\mathrm{C} 1 & 1.3(3) \\ \mathrm{C} 8-\mathrm{O} 1-\mathrm{C} 7-\mathrm{C} 4 & 76.5(2) \\ \mathrm{C} 8-\mathrm{O} 1-\mathrm{C} 7-\mathrm{C} 11 & -163.30(17) \\ \mathrm{C} 3-\mathrm{C} 4-\mathrm{C} 7-\mathrm{O} 1 & -130.98(19) \\ \mathrm{C} 5-\mathrm{C} 4-\mathrm{C} 7-\mathrm{O} 1 & 49.5(2) \\ \mathrm{C} 3-\mathrm{C} 4-\mathrm{C} 7-\mathrm{C} 11 & 108.0(2) \\ \mathrm{C} 5-\mathrm{C} 4-\mathrm{C} 7-\mathrm{C} 11 & -71.5(2) \\ \mathrm{C} 7-\mathrm{O} 1-\mathrm{C} 8-\mathrm{C} 9 & 65.9(2) \\ \mathrm{C} 12-\mathrm{N} 1-\mathrm{C} 11-\mathrm{O} 2 & 1.5(3) \\ \mathrm{C} 12-\mathrm{N} 1-\mathrm{C} 11-\mathrm{C} 7 & -177.93(19) \\ \mathrm{O} 1-\mathrm{C} 7-\mathrm{C} 11-\mathrm{O} 2 & 161.04(17) \\ \mathrm{C} 4-\mathrm{C} 7-\mathrm{C} 11-\mathrm{O} 2 & -77.0(2) \\ \mathrm{O} 1-\mathrm{C} 7-\mathrm{C} 11-\mathrm{N} 1 & -19.5(3) \\ \mathrm{C} 4-\mathrm{C} 7-\mathrm{C} 11-\mathrm{N} 1 & 102.5(2)\end{array}$

$\mathrm{C} 19-\mathrm{C} 14-\mathrm{C} 15-\mathrm{C} 16$

$\mathrm{C} 13-\mathrm{C} 14-\mathrm{C} 15-\mathrm{C} 16$

$\mathrm{C} 20-\mathrm{O} 3-\mathrm{C} 16-\mathrm{C} 15$

$\mathrm{C} 20-\mathrm{O} 3-\mathrm{C} 16-\mathrm{C} 17$

$\mathrm{C} 14-\mathrm{C} 15-\mathrm{C} 16-\mathrm{O} 3$

$\mathrm{C} 14-\mathrm{C} 15-\mathrm{C} 16-\mathrm{C} 17$

$\mathrm{C} 21-\mathrm{O} 4-\mathrm{C} 17-\mathrm{C} 18$

$\mathrm{C} 21-\mathrm{O} 4-\mathrm{C} 17-\mathrm{C} 16$

$\mathrm{O} 3-\mathrm{C} 16-\mathrm{C} 17-\mathrm{O} 4$

$\mathrm{C} 15-\mathrm{C} 16-\mathrm{C} 17-\mathrm{O} 4$

$\mathrm{O} 3-\mathrm{C} 16-\mathrm{C} 17-\mathrm{C} 18$

$\mathrm{C} 15-\mathrm{C} 16-\mathrm{C} 17-\mathrm{C} 18$

$\mathrm{O} 4-\mathrm{C} 17-\mathrm{C} 18-\mathrm{C} 19$

C16-C17-C18-C19

$\mathrm{C} 15-\mathrm{C} 14-\mathrm{C} 19-\mathrm{C} 18$

C13-C14-C19-C18

$\mathrm{C} 17-\mathrm{C} 18-\mathrm{C} 19-\mathrm{C} 14$

$\mathrm{C} 17-\mathrm{O} 4-\mathrm{C} 21-\mathrm{C} 22$
$-1.2(3)$

$-178.71(19)$

$-3.9(3)$

176.77 (19)

$-178.8(2)$

$0.5(3)$

$5.6(3)$

$-175.78(18)$

$1.2(3)$

$-178.20(18)$

$179.9(2)$

$0.5(3)$

$177.8(2)$

-0.8 (3)

$1.0(3)$

$178.4(2)$

$0.0(3)$

$-69.6(3)$

Hydrogen-bond geometry $\left(\AA,{ }^{\circ}\right)$

$\mathrm{Cg} 2$ is the centroid of the $\mathrm{C} 14-\mathrm{C} 19$ ring.

\begin{tabular}{lllll}
\hline$D-\mathrm{H} \cdots A$ & $D-\mathrm{H}$ & $\mathrm{H} \cdots A$ & $D \cdots A$ & $D-\mathrm{H} \cdots A$ \\
\hline $\mathrm{N} 1-\mathrm{H} 1 N \cdots \mathrm{O} 2^{\mathrm{i}}$ & 0.88 & 2.04 & $2.850(2)$ & 152 \\
$\mathrm{C} 10-\mathrm{H} 10 \cdots \mathrm{O} 2^{\mathrm{ii}}$ & 0.95 & 2.35 & $3.218(3)$ & 152 \\
$\mathrm{C} 12-\mathrm{H} 12 A \cdots \mathrm{O} 1^{\mathrm{iii}}$ & 0.99 & 2.53 & $3.232(3)$ & 128 \\
$\mathrm{C} 20-\mathrm{H} 20 C \cdots \mathrm{Cg} 2^{\mathrm{iv}}$ & 0.98 & 2.84 & $3.709(3)$ & 148 \\
\hline
\end{tabular}

Symmetry codes: (i) $x,-y+1 / 2, z-1 / 2$; (ii) $x,-y+3 / 2, z-1 / 2$; (iii) $x,-y+1 / 2, z+1 / 2$; (iv) $x,-y-1 / 2, z-1 / 2$. 